\title{
Hepatocellular Carcinoma with Irregular Rim-Like Arterial Phase Hyperenhancement: More Aggressive Pathologic Features
}

\author{
Hyungjin Rhee $^{a}$ Chansik An $^{a}$ Hye-Young Kim ${ }^{b}$ Jeong Eun Yoo ${ }^{b}$ \\ Young Nyun Park ${ }^{b}, c$ Myeong-Jin Kim ${ }^{a}$ \\ a Department of Radiology, Research Institute of Radiological Science, Severance Hospital, \\ Yonsei University College of Medicine, Seoul, South Korea; ${ }^{\mathrm{b}}$ Department of Pathology, \\ Brain Korea 21 PLUS Project for Medical Science, Integrated Genomic Research Center \\ for Metabolic Regulation, Yonsei University College of Medicine, Seoul, South Korea; \\ 'Severance Biomedical Science Institute, Yonsei University College of Medicine, Seoul, \\ South Korea
}

\section{Keywords}

Fibrous stroma - Hypoxia - Stemness - Macrotrabecular pattern - Microvascular density

\begin{abstract}
Background and Aims: The purpose of our study was to examine the histopathologic characteristics of hepatocellular carcinoma (HCC) with irregular rim-like arterial phase enhancement (IRE), which has been reported to be associated with more aggressive tumor behavior. Methods: We investigated 84 pathologically confirmed HCCs in 84 patients who underwent curative hepatic resection after gadoxetate-enhanced magnetic resonance imaging between January 2008 and February 2013. Two abdominal radiologists independently reviewed these images and classified HCCs into two categories: HCC showing IRE (IRE-HCC) and HCC showing hypoenhancement or diffuse arterial enhancement (non-IRE-HCC). Twenty-two HCCS were classified as IRE-HCCs, and 51 were classified as non-IRE-HCCs concordantly by both reviewers. The remaining $11 \mathrm{HCCs}$, on whose radiologic classifications the reviewers disagreed, were classified as HCCs with intermediate enhancement patterns. The HCC clinicopathologic characteristics and patient outcomes were then compared. Results: IRE-HCCS showed more frequent microvascular invasion (91 vs. 35\%), lower microvascular density (246.5 vs. $426.5 / \mathrm{mm}^{2}$ ), higher proportions of sinusoid-like microvascular pattern ( $55 \mathrm{vs} .0 \%$ ) and macrotrabecular pattern (45 vs. $0 \%$ ), and larger areas of tumor necrosis (15 vs. $0 \%$ ) and fibrous stroma (8.3 vs. $2.1 \%$ ) than non-IRE-HCCs. IRE-HCCs also expressed higher levels of immuno-
\end{abstract}

Hyungjin Rhee and Chansik An contributed equally to this work. 


\section{Liver Cancer}

markers of hypoxia (carbonic anhydrase IX, 64 vs. $8 \%$ ) and stemness (EpCAM, 50 vs. 20\%). $p$ values were $<0.001$ for all comparisons except for EpCAM $(p=0.026)$. HCCs with intermediate enhancement patterns showed mixed/intermediate pathologic features from both IRE- and non-IRE-HCCs. IRE-HCC patients showed poorer 5-year disease-free survival after curative resection than non-IRE-HCC patients $(p=0.005)$. Conclusions: IRE-HCCs demonstrate aggressive histopathologic features, including more hypoxic and fibrotic tumor microenvironments and increased stemness, compared to non-IRE-HCCs. IRE might therefore serve as a noninvasive imaging biomarker for aggressive HCC.

(c) 2018 S. Karger AG, Base

\section{Introduction}

Most hepatocellular carcinomas (HCCs) are hypervascular, and usually exhibit diffuse or global hyperenhancement in the arterial phase of dynamic contrast-enhanced computed tomography or magnetic resonance imaging (MRI), whether it be homogeneous or heterogeneous $[1,2]$. However, some HCCs display an atypical enhancement pattern of peripheral irregular rim-like enhancement (IRE) with central hypoenhancing areas in the arterial phase. HCCs with such IRE patterns (IRE-HCCs) were reported in 5-13\% of patients undergoing curative resection or radiofrequency ablation [3-5]. The IRE pattern is not a typical imaging feature of HCC; it is a finding usually indicative of other hepatic malignancies such as cholangiocarcinoma or combined hepatocellular-cholangiocarcinoma, which generally show worse prognoses than HCC $[6,7]$. In patients with chronic liver disease, however, a significant proportion of hepatic tumors showing IRE are HCCs, because the incidence of this malignancy is much higher than that of other malignancies with underlying liver diseases $[8,9]$.

Several studies have demonstrated that IRE-HCCs show more rapid progression and worse prognosis after curative resection, radiofrequency ablation, or transarterial chemoembolization compared to non-IRE-HCCs [3-5, 10-12]. The unique radiologic features of IRE-HCCs suggest that they might be associated with distinctive histopathologic characteristics responsible for their aggressive tumor behavior. If there are evident associations between IRE and prognostic histopathologic features, IRE could be used as an imaging biomarker for prognosis based on the associations. However, to the best of our knowledge, no comprehensive studies of the histopathologic features of IRE-HCCs exist, although some histopathologic findings observed in IRE-HCCs have been sporadically described [13-15]. Therefore, the purpose of this study was to examine the histopathologic characteristics of HCCs showing IRE in the arterial phase of gadoxetate-enhanced MRI, and compare them to HCCs without IRE.

\section{Materials and Methods}

\section{Subjects}

This retrospective study was approved by our institutional review board, and the requirement for patient consent was waived. We searched our institution's database and identified 631 patients with chronic liver disease in whom HCC was histologically diagnosed after hepatic resection between January 2008 and February 2013. Among these patients, we excluded 186 in whom hepatic resection was not performed with curative intent $(n=87)$, gadoxetate-enhanced MRI was not performed within 3 months before surgery $(n=$ 70), evaluation of preoperative MRI was not possible due to antecedent locoregional treatment $(n=29)$, or diffuse infiltrative HCCs $(n=11)$ or HCCs in dysplastic nodules $(n=3)$ were present. The remaining 431 patients were included in the study. The MRI data, surgical notes, and pathology reports of these patients were retrospectively reviewed by a board-certified abdominal radiologist (reviewer 1, C.A.) with 6 years of experience in liver MRI, who then identified 470 histologically confirmed HCCs, and classified the largest HCC 


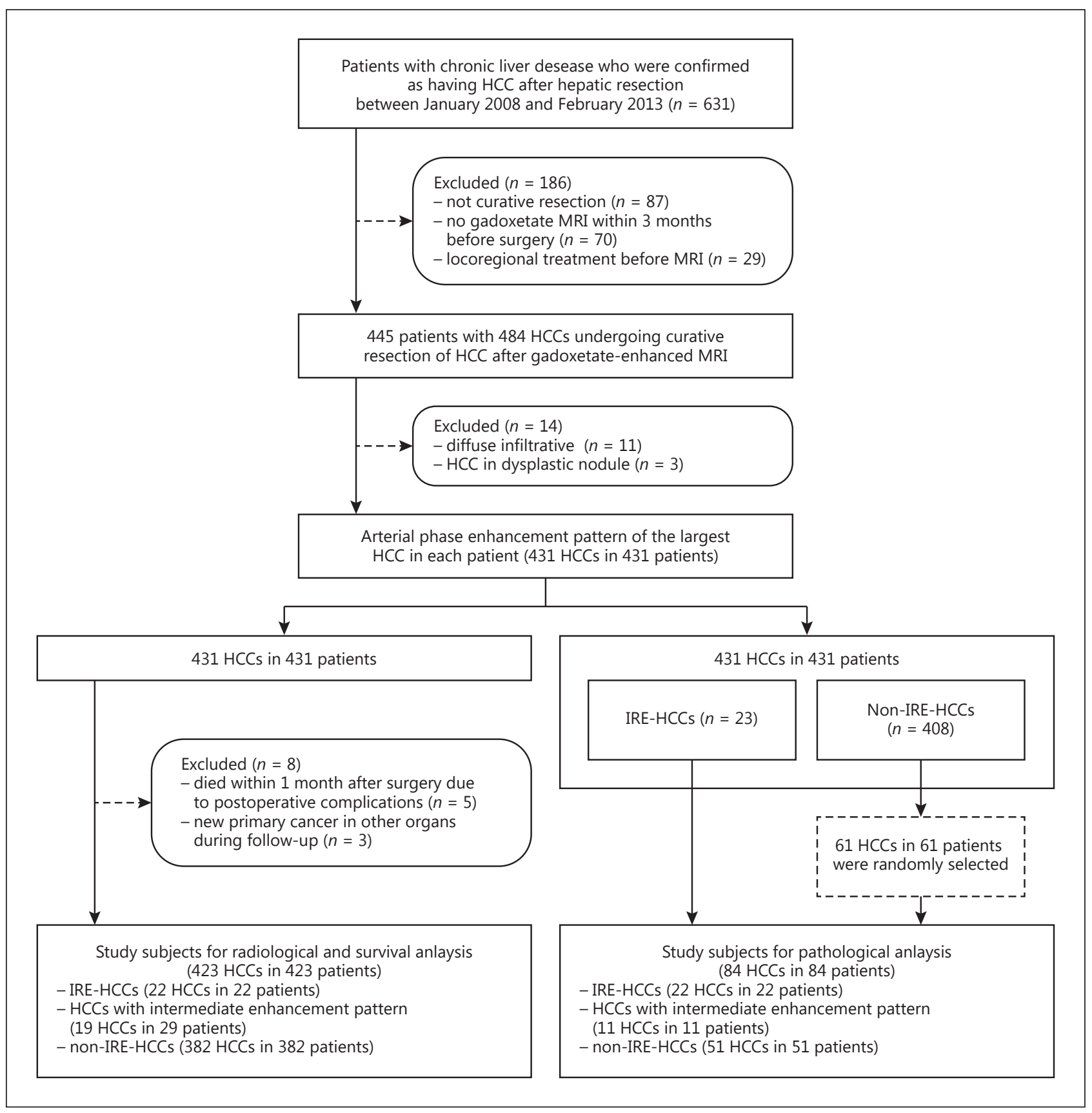

Fig. 1. Flow diagram of this study. HCC, hepatocellular carcinoma; MRI, magnetic resonance imaging; IRE, irregular rim-like enhancement.

in each patient into one of two types based on their arterial phase enhancement pattern: IRE-HCC and nonIRE-HCC (see Arterial Phase Enhancement Pattern below). All the 23 IRE-HCCs in 23 patients were included in the study. As a control group, 61 patients were randomly selected among those with non-IRE-HCCs. Therefore, 84 patients with 84 HCCs comprised our final study subjects (Fig. 1).

To examine the inter-reader variability and its impacts on study results, another abdominal radiologist with 23 years of experience in liver MRI (reviewer 2, M.-J.K.) reviewed the MRI images of our initial study population and classified all the 431 HCCs into IRE-HCCs vs. non-IRE-HCCs. During the image analysis, both 


\section{Liver Cancer}

\begin{tabular}{l|l}
\hline Liver Cancer 2019;8:24-40 \\
\hline DOI: 10.1159/000488540 & $\begin{array}{l}\text { @ 2018 S. Karger AG, Basel } \\
\text { www.karger.com/lic }\end{array}$ \\
\hline
\end{tabular}
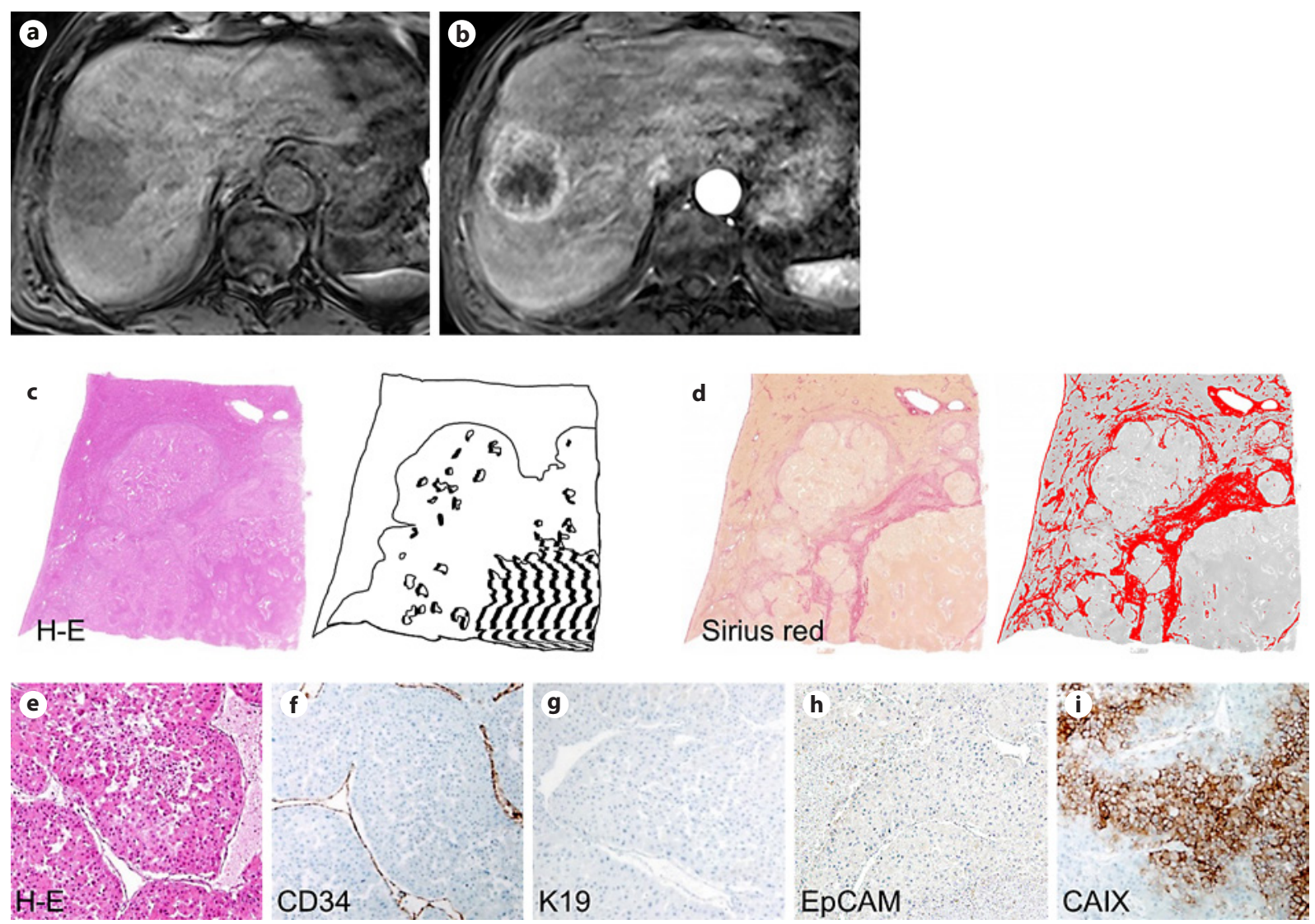

Fig. 2. A 71-year-old male hepatitis B virus carrier with hepatocellular carcinoma exhibiting irregular rimlike enhancement (IRE-HCC). A 5.5-cm tumor in the right superior hepatic section shows low-signal intensity on precontrast T1-weighted imaging (a) as well as IRE in the arterial phase (b) of gadoxetate-enhanced MRI. Tumor necrosis is more prominent in the central portion of HCC (hatched in black) (c, hematoxylineosin [H-E] stain), and fibrous tumor stroma is marked by a red color (d, Sirius red stain). The tumor mainly comprises a macrotrabecular pattern (e, H-E stain). A sinusoid-like microvascular pattern is noted with low microvascular density as $177 / \mathrm{mm}^{2}$ on immunostaining for CD34 (f), K19 (g) and EpCAM (h) are not expressed, while positive expression of carbonic anhydrase IX (CAIX) is noted (i). Original magnification, $\times 100$.

radiologists were aware that all the tumors were histologically confirmed HCCs but were blinded to information about the patients' clinical outcomes and the tumors' histopathologic features.

Clinical information and laboratory data of those patients were retrospectively reviewed by an investigator who did not participate in the image analysis (H.R.), including patient demographics and survival data, the cause of chronic liver disease, serum levels of aspartate transaminase, alanine transaminase, albumin, platelets, $\alpha$-fetoprotein, and protein induced by vitamin $\mathrm{K}$ absence (PIVKA)-II, and Child-Pugh class. The disease-free survival rate was calculated from the date of the initial treatment to either the date of death or the last follow-up visit before December 27, 2017.

\section{Dynamic Contrast-Enhanced MRI}

MRI was performed using two 3.0-T systems (MAGNETOM Trio a Tim; Siemens Medical Solutions, Erlangen, Germany; Intera Achieva, Philips Medical Systems, Best, The Netherlands) or a 1.5-T system (Intera Achieva, Philips Medical Systems). For dynamic imaging, $10 \mathrm{~mL}$ of gadoxetic acid disodium (Primovist; Bayer Schering Pharma, Berlin, Germany) was injected followed by $20 \mathrm{~mL}$ of $0.9 \%$ saline at an injection rate of 1 or $2 \mathrm{~mL} / \mathrm{s}$. Three-dimensional gradient echo sequences with chemical-selective fat suppression were acquired 


\section{Liver Cancer}

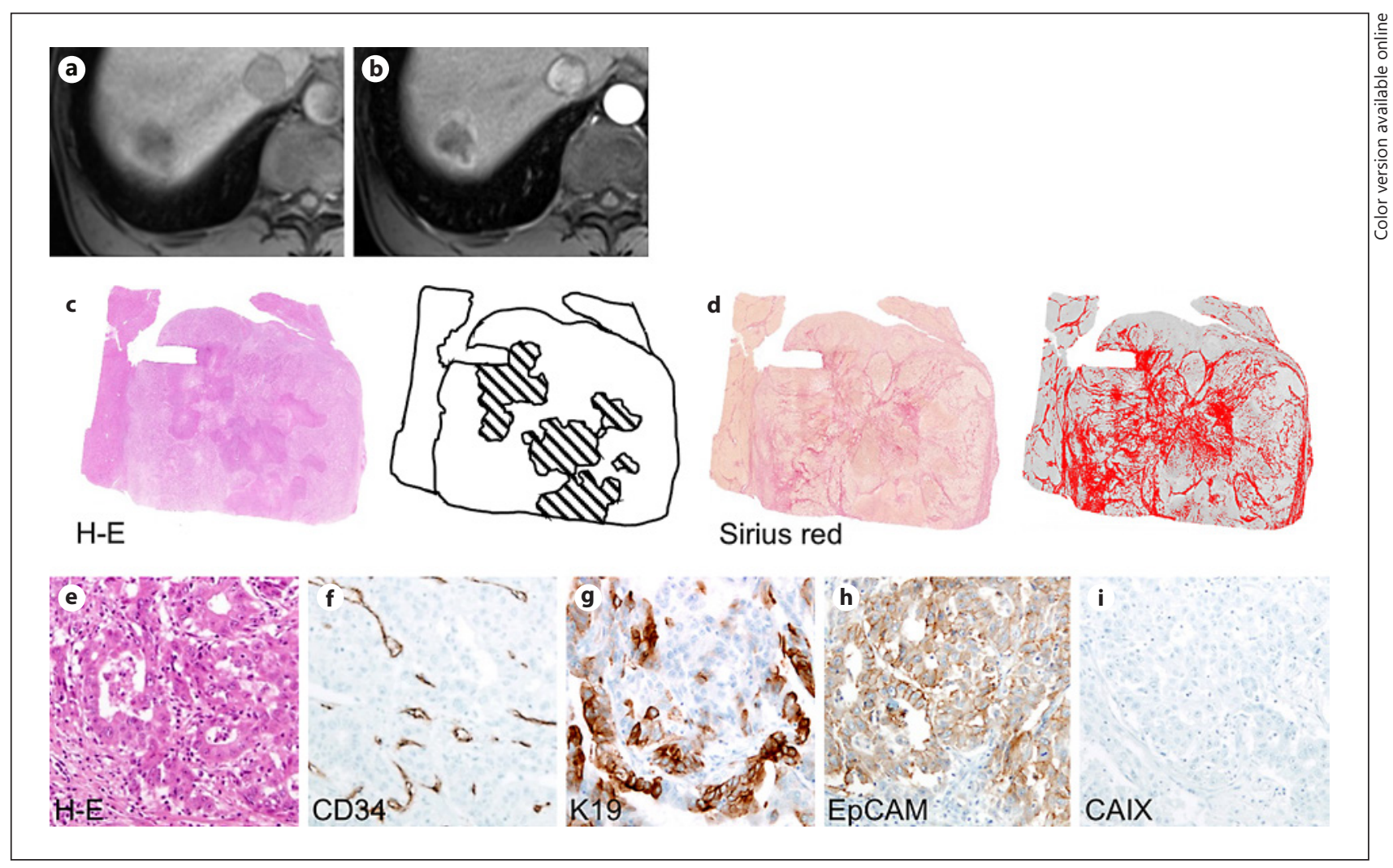

Fig. 3. A 73-year-old male with hepatitis B virus-related cirrhosis and hepatocellular carcinoma with irregular rim-like enhancement (IRE-HCC). A 3-cm tumor in segment 8 of the liver shows low-signal intensity on precontrast T1-weighted imaging (a) and IRE in the arterial phase (b) of gadoxetate-enhanced MRI. There is necrosis in its central portion (c) (hematoxylin-eosin [H-E] stain, necrosis is hatched in black), and a high proportion of fibrosis is present (d) (Sirius red stain; fibrous stroma is colored in red). On histopathologic analysis, HCC demonstrated abundant fibrous stroma-intervening cords of tumor cells (e, H-E stain), mixed sinusoid-like and capillary-like microvascular pattern with low microvascular density (199/ $\mathrm{mm}^{2}$ ) (f), positive expression of K19 (g) and EpCAM (h), and negative expression of carbonic anhydrase IX (CAIX) (i). Original magnification, $\times 200$.

before and after intravenous injection of the contrast agent, using a section thickness, repetition time, and echo time of 2-4 mm, 2.54-4.48 ms, and $0.92-2.2 \mathrm{~ms}$, respectively. After obtaining the precontrast images, four consecutive contrast-enhanced dynamic images were acquired: arterial, early portal, late portal, and late dynamic phases approximately 25-30, 65-75, 95-105, and 130-150 s after the contrast injection, respectively.

\section{Arterial Phase Enhancement Pattern}

The arterial phase contrast-enhancement pattern was classified into either IRE or non-IRE. IRE was defined as irregularly shaped rim-like peripheral hyperenhancement with central hypoenhancing areas (Fig. 2, 3) [4]. Diffuse/global enhancement (defined as enhancement that unequivocally is greater than that of the liver and is distributed over a large area) or hypoenhancement (defined as enhancement that is similar to or less than that of the liver) was considered non-IRE (Fig. 4) [16, 17]. HCCs with heterogeneous enhancement without apparent rim-like pattern were also considered as non-IRE.

Histopathology and Immunohistochemistry

Histopathologic data including tumor diameter, differentiation (Edmondson-Steiner grade), capsule formation status, microvascular invasion, tumor multiplicity, and the proportion of the tumor's necrotic area 


\section{Liver Cancer}
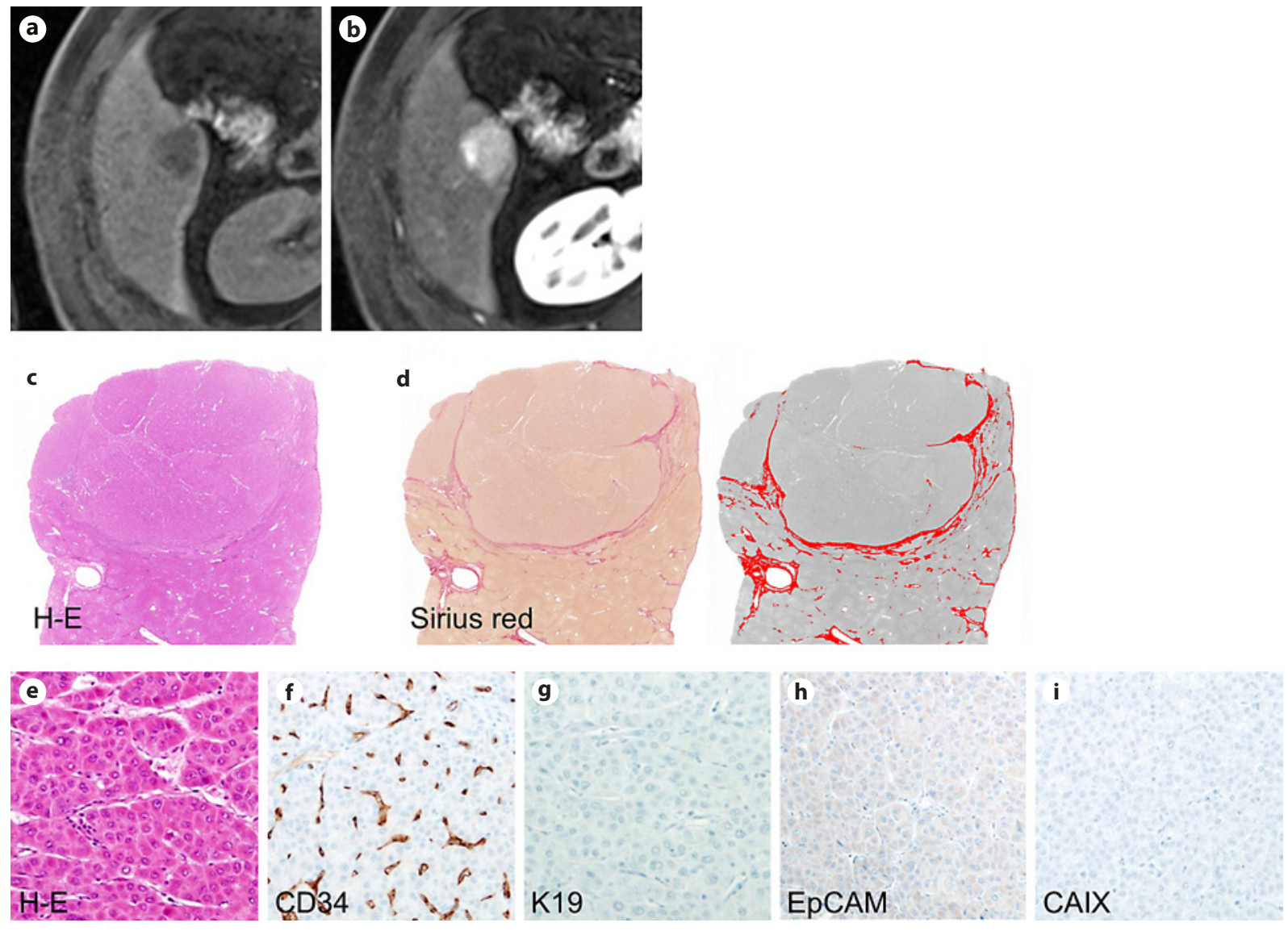

Fig. 4. A 37-year-old male hepatitis B virus carrier with hepatocellular carcinoma lacking irregular rim-like enhancement (non-IRE-HCC). A 2.2-cm tumor in the right liver exhibited low-signal intensity on precontrast T1-weighted imaging (a) and diffuse enhancement in the arterial phase (b) of gadoxetate-enhanced MRI. There is no necrotic area in HCC on the hematoxylin-eosin [H-E] stain (c). The fibrous tumor stroma was stained with Sirius red, and its proportions were then marked with a red color and quantitated using ImageJ software. The proportion of fibrous stroma, excluding the tumor capsule, was less than $1 \%$. (d). No areas with macrotrabecular patterns were observed (e). A capillary-like microvascular pattern and high microvascular density $\left(438 / \mathrm{mm}^{2}\right)$ are noted on immunostaining for CD34 (f), and K19 (g), EpCAM (h), and carbonic anhydrase IX (CAIX) (i) are not expressed. Original magnification, $\times 200$.

were retrospectively collected from the pathology reports. In cases of multiple tumors, all histopathologic analyses were performed on the largest lesion of each patient; this was the same lesion in which arterial enhancement pattern classification was performed. We additionally evaluated histopathologic characteristics and tumor microenvironmental factors on a representative whole-section slide, as follows: a macrotrabecular pattern was defined as cords of tumor cells thicker than 10 cells, as previously described [18]. The proportion of the tumor area showing a macrotrabecular pattern was recorded, and tumors with $>50 \%$ of their areas exhibiting macrotrabecular patterns were defined as macrotrabecular-massive type [19]. To assess the proportion of fibrous stroma of a tumor, the whole-section slide of each sample was stained with Sirius red and digitized with a high-resolution slide scanner (LS-4000ed; Nikon Corporation, Tokyo, Japan) [20].

Considering that IRE-HCCs have internal hypoenhancing areas, we hypothesized that they might have a distinct microvascular pattern with an internal hypoxic tumor microenvironment. Therefore, using immunohistochemical staining, we investigated the microvascular pattern with the widely used endothelial cell 


\section{Liver Cancer}

marker, CD34 (1:100; DAKO, Glostrup, Denmark) [21] and the presence of a hypoxic microenvironment with a hypoxia marker, carbonic anhydrase IX (CAIX; 1:1,000; Abcam, Cambridge, MA, USA) [22]. In addition, we studied the expression of stemness markers which are known to be associated with hypoxic microenvironment and aggressive biologic behavior of HCC: keratin 19 (K19; 1:100; DAKO) and epithelial cell adhesion molecule (EpCAM; 1:1,000; Calbiochem, Darmstadt, Germany) [23]. Immunohistochemical staining was performed using an automated staining system (Discovery XT; Ventana Medical Systems, AZ, USA), and the slides were counterstained with hematoxylin.

To study the characteristics of the microvasculature in HCCs, the microvascular patterns and densities were evaluated using CD34 immunostaining $[24,25]$. The microvascular patterns in HCCs were categorized as follows: (1) capillary-like microvascular pattern showing small and scattered capillaries with little or narrow lumens, and (2) sinusoid-like microvascular pattern showing a continuous and branching vascular structure with an open sinusoidal space [24, 25]. In cases where both patterns were observed (mixed cases), the proportion of each pattern was recorded. The microvascular density was evaluated in five randomly selected high-power fields (200× magnification; one high-power field corresponds to $0.95 \mathrm{~mm}^{2}$ ) as follows: for the capillary-like microvascular pattern, one capillary corresponded to 1 point of microvessel; for the sinusoid-like microvascular pattern, every $40 \mu \mathrm{m}$ of endothelial-lined sinusoid counted toward 1 point of microvessel [24]. Large vessels with thick muscular walls were excluded.

Positivity for K19, EpCAM, and CAIX was defined as follows: for K19, the cytoplasmic expression in $\geq 5 \%$ of tumor cells with moderate or strong intensity; for EpCAM and CAIX, the membranous expression in $\geq 5 \%$ of tumor cells with moderate or strong intensity. The histopathology and immunohistochemically stained slides were reviewed by two readers in consensus (Y.N.P., a pathologist, and H.R., a radiologist, with $>15$ and 5 years of experience in liver pathology, respectively) who were blinded to the radio-clinical information. The proportion of fibrous stroma was evaluated independently, and the mean value of the two readings was used for subsequent analyses.

\section{Statistical Analysis}

Inter-reader agreement was expressed by Cohen's kappa coefficient. A kappa statistic of 0.8-1.0 was considered excellent agreement, 0.6-0.79 good agreement, 0.40-0.59 moderate agreement, 0.2-0.39 fair agreement, and 0-0.19 poor agreement. To compare features of HCCs with and without IRE, we used the Mann-Whitney U test for continuous variables and the $\chi^{2}$ or Fisher exact test for categorical variables. For survival analysis, our initial study subjects of 431 patients (prior to the random selection of 61 patients with non-IRE-HCCs) were included. The endpoint for survival analysis was disease-free survival; hence, all HCCrelated deaths and recurrences were considered relevant events. Living patients without recurrence were censored on the date of the last follow-up. The Kaplan-Meier method was used to calculate disease-free survival rates, and differences were compared using the log-rank test. The correlation analysis was performed by calculating Pearson's correlation coefficients and $p$ values. Bonferroni correction was used for post hoc multiple comparisons for all statistical analyses. Two-sided $p$ values $<0.05$ were considered statistically significant. All statistical analyses were performed using R software (version 3.4.0; The R Foundation for Statistical Computing, Vienna, Austria).

\section{Results}

\section{Radiologic Classification and Inter-Reader Agreement}

Of our final 84 HCCs selected for histopathologic analysis, 23 (27\%) and 61 (73\%) were classified as IRE-HCCs and non-IRE-HCCs by reviewer 1, respectively. Among the same 84 HCCs, 32 (38\%) and 52 (62\%) were classified as IRE-HCCs and non-IRE-HCCs by reviewer 2, respectively. To reconcile the results of the two reviewers, HCCs were divided into three categories: (1) IRE-HCCs, which both reviewers agreed exhibit IRE, (2) HCCs with intermediate enhancement patterns, which only one of the two reviewers deemed exhibit IRE, and (3) nonIRE-HCCs, which both reviewers agreed show no IRE. Following this classification, the numbers of IRE-HCCs, HCCs with intermediate enhancement patterns, and non-IRE-HCCs were 22 (26\%), $11(13 \%)$, and 51 (61\%), respectively. 
Table 1. Baseline characteristics of the included patients

\begin{tabular}{|c|c|c|c|c|c|}
\hline Variables & $\begin{array}{l}\text { Total } \\
(n=84,100 \%)\end{array}$ & $\begin{array}{l}\text { IRE-HCCs } \\
(n=22,26 \%)\end{array}$ & $\begin{array}{l}\text { HCCs with intermediate } \\
\text { enhancement patterns } \\
(n=11,13 \%)\end{array}$ & $\begin{array}{l}\text { Non-IRE-HCCs } \\
(n=51,61 \%)\end{array}$ & $p$ value \\
\hline Age, years & $56.1 \pm 9.6$ & $54.5 \pm 9.3$ & $58.0 \pm 6.4$ & $56.4 \pm 10.3$ & 0.597 \\
\hline Sex & & & & & 0.490 \\
\hline Male & 65 (77) & $18(82)$ & $7(64)$ & $40(78)$ & \\
\hline Female & $19(23)$ & 4 (18) & $4(36)$ & $11(22)$ & \\
\hline Etiology & & & & & 0.243 \\
\hline Hepatitis B & $70(83)$ & $18(82)$ & $11(100)$ & $41(80)$ & \\
\hline Hepatitis C & $2(2)$ & $0(0)$ & $0(0)$ & $2(4)$ & \\
\hline Alcohol & $10(12)$ & $2(9)$ & $0(0)$ & $8(16)$ & \\
\hline Unknown & $2(2)$ & $2(9)$ & $0(0)$ & $0(0)$ & \\
\hline \multicolumn{6}{|l|}{ Child-Pugh class } \\
\hline A & $82(98)$ & $21(95)$ & $11(100)$ & $50(98)$ & 0.634 \\
\hline B & $2(2)$ & $1(5)$ & $0(0)$ & $1(2)$ & \\
\hline Cirrhosis & $44(52)$ & $12(55)$ & $8(73)$ & $24(47)$ & 0.294 \\
\hline AST, IU/L & $32(25-42)$ & $30(23-33)$ & $40(24-43)$ & $32(25-45)$ & 0.491 \\
\hline ALT, IU/L & $29(22-42)$ & $30(24-38)$ & $30(23-38)$ & 27 (20-48) & 0.941 \\
\hline Albumin, g/dL & $4.3(4.1-4.6)$ & $4.3(3.9-4.5)$ & $4.4(4.2-4.5)$ & $4.3(4.1-4.6)$ & 0.631 \\
\hline Platelets, $1,000 / \mu \mathrm{L}$ & $172.9 \pm 57.5$ & $184.6 \pm 61.4$ & $184.7 \pm 49.2$ & $165.4 \pm 57.4$ & 0.328 \\
\hline $\mathrm{AFP}, \mathrm{IU} / \mathrm{mL}$ & $24.1(4.0-197.3)$ & $40.0(5.8-178.4)$ & $41.9(5.1-647.8)$ & $13.5(3.9-60.1)$ & 0.283 \\
\hline PIVKA-II', AU/mL & $79.0(36.0-300.0)$ & $241.5(87.5-1064.0)$ & $42.0(27.5-118.5)$ & $59.5(34.5-186.5)$ & 0.013 \\
\hline Tumor multiplicity & & & & & 0.157 \\
\hline Solitary & 75 (89) & $18(82)$ & $9(82)$ & $48(94)$ & \\
\hline Two or three & $9(11)$ & $4(18)$ & $2(18)$ & $3(6)$ & \\
\hline
\end{tabular}

Numerical variables are expressed as median (interquartile range) or mean \pm standard deviation, according to the result of the normality test (Shapiro-Wilktest). Categorical variablesare expressedas $n(\%)$. IRE, irregular rim-like enhancement; HCC, hepatocellular carcinoma; AST, aspartate transaminase; ALT, alanine transaminase; AFP, $\alpha$-fetoprotein; PIVKA, protein induced by vitamin Kabsence. a Serum PIVKA-II level was not available for 1 patient. In post hoc analysis with Bonferroni correction, PIVKA-II level was significantly higher in IRE-HCCs than non-IRE HCCs $(p=0.048)$ or HCCs with intermediate patterns $(p=0.039)$.

In our initial study population of 431 patients (prior to the random selection of 61 nonIRE-HCCs), reviewer 1 and reviewer 2 classified 23 (5\%) and 40 (9\%) as having IRE-HCCs, respectively. Combining their results, the numbers of IRE-HCCs, HCCs with intermediate enhancement patterns, and non-IRE-HCCs were 22 (5\%), 19 (4\%), and 390 (91\%), respectively. The inter-reader agreement for classification of IRE-HCCs and non-IRE-HCCs was good, with a kappa coefficient of 0.676 .

\section{Baseline Clinical Characteristics of Patients}

The patient characteristics are summarized in Table 1. Our 84 patients comprised 65 males and 19 females with a median age of 55 years. Chronic hepatitis B viral infection was the most predominant cause of underlying liver disease (83\% of patients), and $52 \%$ had cirrhosis. Most patients (98\%) were of Child-Pugh class A. Of the 84 patients, 75 (89\%) had solitary HCCs, while $9(11 \%)$ had multiple (2 or 3) HCCs. Except for serum PIVKA-II level, which was higher in patients with IRE-HCCs than those with non-IRE-HCCs $(p=0.048)$ or HCCs with intermediate enhancement patterns $(p=0.039)$, there were no significant differences in age, sex, etiology of chronic liver disease, presence/absence of cirrhosis, serum levels of alanine transaminase, aspartate transaminase, albumin, platelets, and $\alpha$-fetoprotein, or tumor multiplicity ( $p>0.157)$. 


\section{Histopathologic Characteristics of HCCs}

We compared the histopathologic characteristics between the three categories (IREHCCs, HCCs with intermediate enhancement patterns, and non-IRE-HCCs) based on the combined results (Table 2; Fig. 5). Upon comparing the IRE-HCCs to non-IRE-HCCs, IRE-HCCs demonstrated a higher proportion of macrotrabecular pattern $(p<0.001)$, more frequent macrotrabecular-massive type $(p<0.001)$, and more frequent microvascular invasion $(p<$ $0.001)$. The microvascular density was lower in IRE-HCCs than in non-IRE-HCCs $(p<0.001)$; for microvascular patterns, IRE-HCCs showed a lower proportion of capillary-like microvascular patterns and a higher proportion of sinusoid-like microvascular patterns $(p<0.001$ for both). IRE-HCCs showed larger areas of tumor necrosis $(p<0.001)$ and fibrous stroma $(p<$ 0.001) compared to non-IRE-HCCs (Fig. 2-4, 5a-e).

We also assessed the correlations between histopathologic factors. The microvascular density was positively correlated with the proportion of capillary-like microvascular pattern, whereas it was negatively correlated with the proportions of sinusoid-like microvascular pattern and macrotrabecular pattern ( $p<0.001$ for all) (Fig. $5 f)$. The proportion of sinusoidlike microvascular pattern was positively correlated with proportions of macrotrabecular pattern and tumor necrosis ( $p<0.005$ for all) (Fig. 5g, h). On immunohistochemical analyses, IRE-HCCs exhibited more frequent expressions of EpCAM $(p=0.026)$ and CAIX $(p<0.001)$ compared to non-IRE-HCCs (Fig. 5i-k). HCCs with intermediate enhancement patterns demonstrated intermediate or mixed pathologic features and immunoprofiles between the concordant cases of IRE-HCCs and non-IRE-HCCs (Fig. 5, 6).

The histopathologic characteristics of IRE-HCCs and non-IRE-HCCs, based on the separate results of the two reviewers' classifications, were also compared and are presented in online supplementary Table 1 and supplementary Figure 1 (for all online suppl. material, see www. karger.com/doi/10.1159/000488540). IRE-HCCs were associated with poorer differentiation, a higher proportion of macrotrabecular pattern, more frequent macrotrabecularmassive type, more frequent microvascular invasion, lower microvascular density, a lower proportion of capillary-like microvascular pattern, a higher proportion of sinusoid-like microvascular pattern, and larger areas of tumor necrosis and fibrous stroma $(p<0.001$ for all according to both reviewers) (see online suppl. Fig. 1A-E). On immunohistochemical analyses, IRE-HCCs showed more frequent expressions of K19, EpCAM, and CAIX compared to non-IRE-HCCs $(p<0.05$ for all according to both reviewers, excluding EpCAM expression for reviewer 2 [ $p=0.055]$ ) (see online suppl. Fig. 1F-H).

\section{Analysis of HCCs with Intermediate Enhancement Patterns}

We further assessed the histopathologic characteristics of the 11 HCCs with intermediate enhancement patterns. Three cases demonstrated a nodule-in-nodule appearance on histopathologic examination, with the outer area showing features typical of non-IRE-HCC (i.e., capillary-like microvascular pattern) and the inner area showing features of IRE-HCC (i.e., sinusoid-like microvascular pattern) (Fig. 6). Among the remaining cases that lacked definite nodule-in-nodule appearances (all but one exhibited at least one histopathologic feature that was characteristic of IRE-HCCs) included low microvascular density (less than 246.5 per $\mathrm{mm}^{2}$, the mean value for IRE-HCCs based on the combined results of the two readers), a high proportion of sinusoid-like microvascular pattern (higher than 55\%, the median value for IRE-HCCs), macrotrabecular-massive type (tumors with a macrotrabecular pattern comprising more than $50 \%$ of the tumor area), a high proportion of tumor necrotic area (higher than 15\%, the median value for IRE-HCCs), or a high proportion of fibrous stromal area (higher than 8.3\%, the median value of IRE-HCCs) (see online suppl. Table 2). 
Rhee et al.: Aggressive Pathologic Features of HCC with Rim-Like Arterial Enhancement

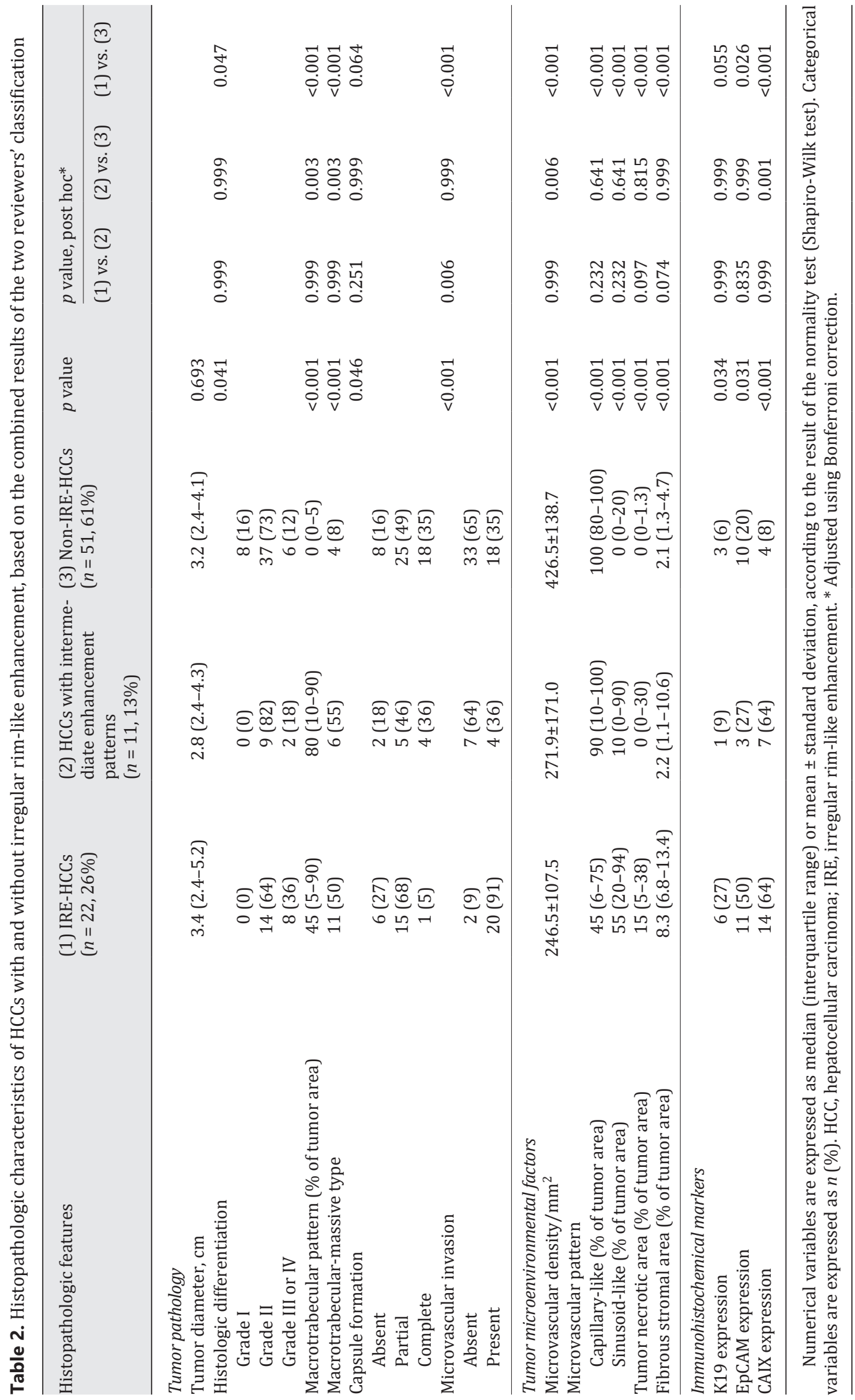




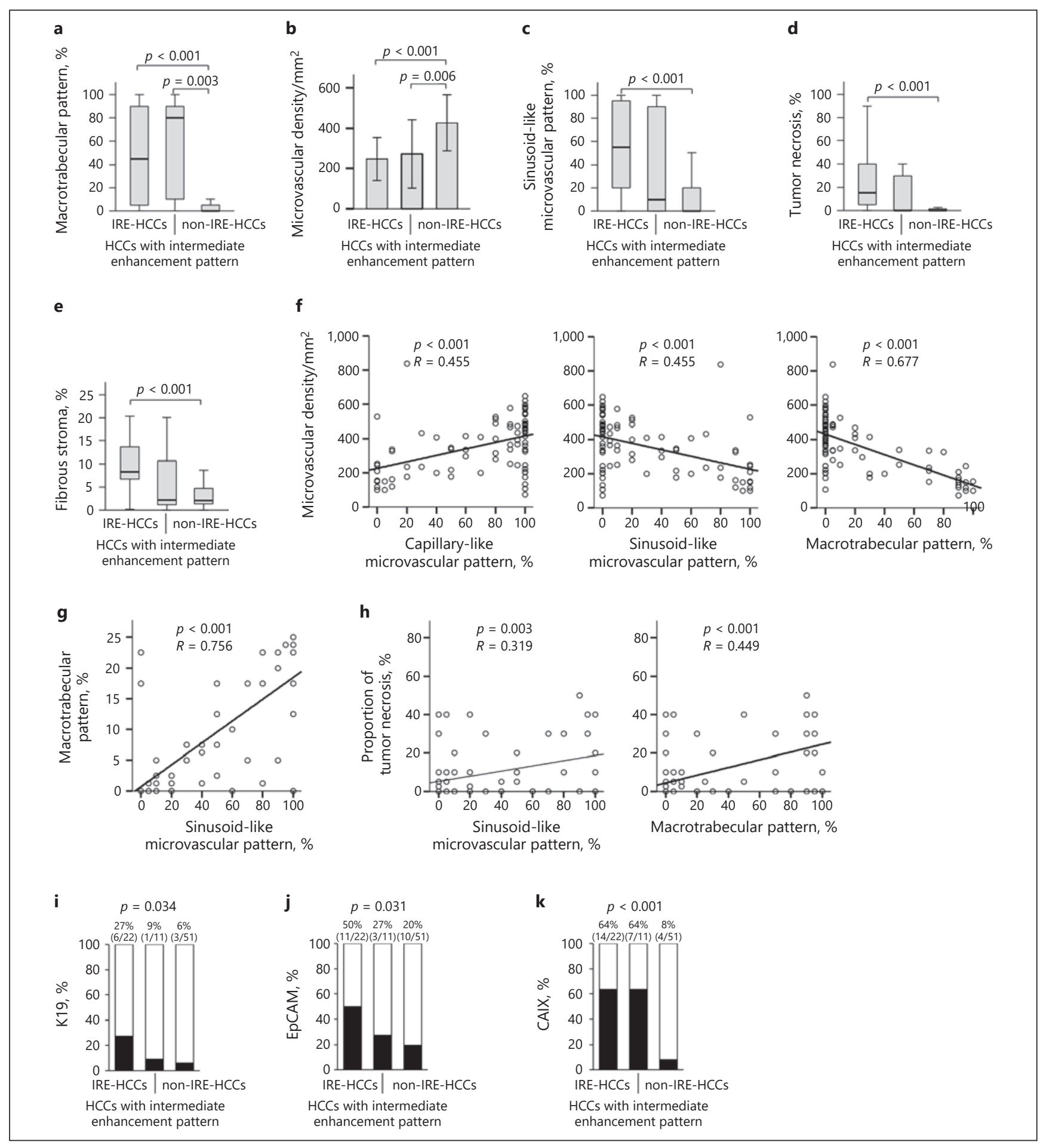

Fig. 5. Comparison of histopathologic characteristics between irregular rim-like enhancement (IRE)-HCCs and non-IRE-HCCs based on the combined results of two reviewers' classifications. Comparison of the proportion of macrotrabecular pattern (a), microvascular density (b), sinusoid-like microvascular pattern (c), tumor necrosis (d), and fibrous stroma (e) between IRE-HCCs, HCCs with intermediate enhancement pattern, and non-IRE-HCCs. $\mathbf{f}-\mathbf{h}$ The correlation between microvascular density, the proportion of capillary-like microvascular pattern, sinusoid-like microvascular pattern, macrotrabecular pattern, and tumor necrosis. Comparison of expression of keratin 19 (K19) (i), epithelial cell adhesion molecule (EpCAM) (j), and carbonic anhydrase IX (CAIX) (k) as assessed by immunohistochemistry in HCCs with intermediate enhancement pattern. 


\section{Liver Cancer}

\begin{tabular}{l|l}
\hline Liver Cancer 2019;8:24-40 \\
\hline DOI: 10.1159/000488540 & $\begin{array}{l}\text { () 2018 S. Karger AG, Basel } \\
\text { www.karger.com/lic }\end{array}$ \\
\hline
\end{tabular}

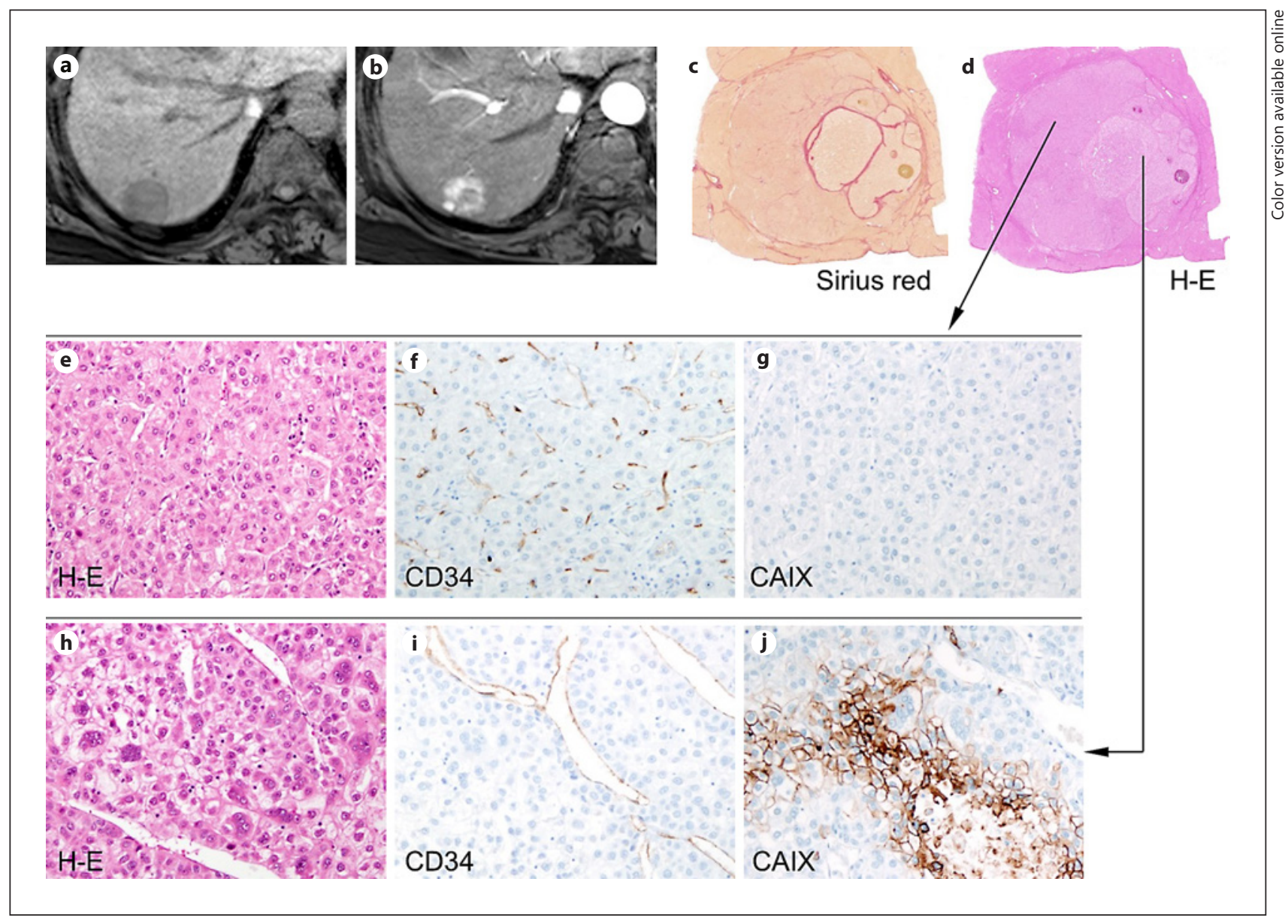

Fig. 6. A 59-year-old female with hepatitis B virus-related cirrhosis and hepatocellular carcinoma (HCC) exhibiting an intermediate enhancement pattern. A 2.7-cm tumor in segment 7 of the liver shows low-signal intensity on precontrast T1-weighted imaging (a). The center of the lesion demonstrates lower arterial enhancement compared to the peripheral portion (b) on gadoxetate-enhanced MRI. On pathologic examination, the HCC shows a nodule-in-nodule pattern (c, Sirius red stain; d, hematoxylin-eosin [H-E] stain). On histopathologic analyses, the outer nodule shows tumor trabeculae with a thickness of $<10$ tumor cells and no macrotrabecular pattern (e, H-E stain), a capillary-like microvascular pattern on immunostaining for CD34 (f), and no carbonic anhydrase IX (CAIX) expression (g). Conversely, the inner nodule exhibits a macrotrabecular pattern (h, H-E stain), a sinusoid-like microvascular pattern on immunostaining for CD34 (i), and is positive for CAIX (j). Original magnification, $\times 200$. K19 is negative in both the inner and outer nodules (data not shown).

\section{Prognosis after HCC Resection}

Of the 431 patients, 8 were excluded from survival analysis because they died within 1 month after surgery due to postoperative complications $(n=5)$ or were found to have new primary cancer in other organs during follow-up $(n=3)$. Prognosis after hepatic resection was worse in patients with IRE-HCC. Based on the reviewers' combined results, the 5-year disease-free survival rates of patients with IRE-HCCs, HCCs with intermediate enhancement patterns, and non-IRE-HCCs were 34, 45, and 63\%, respectively; the difference in prognosis was only statistically significant between the patients with IRE-HCCs and non-IRE-HCCs ( $p=$ 0.005; Fig. 7a). The 5-year disease-free survival rates of patients with IRE-HCCs and non-IREHCCs were 37 and $62 \%$ based on the classifications of reviewer $1(p=0.002)$, and 36 and $63 \%$ 


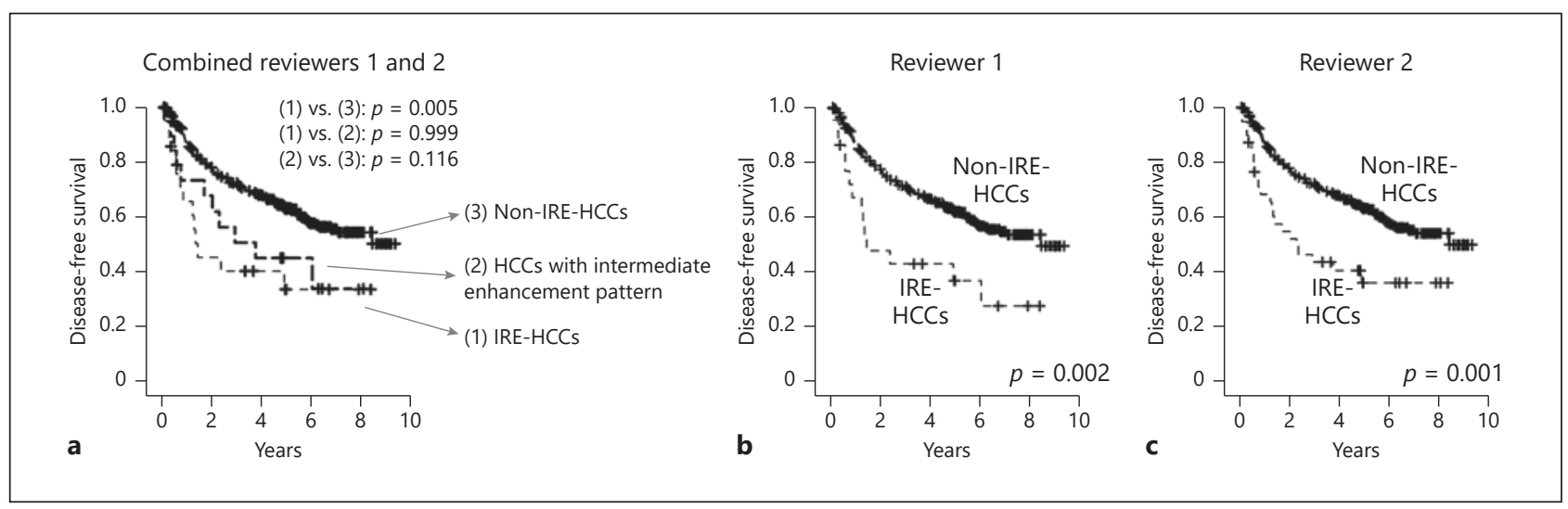

Fig. 7. Comparison of disease-free survival between irregular rim-like enhancement hepatocellular carcinomas (IRE-HCCs) and non-IRE-HCCs. Kaplan-Meier plot for disease-free survival according to the combined classifications of reviewers 1 and 2 (a), reviewer 1 alone (b), and reviewer 2 alone (c).

based on the classifications of reviewer $2(p=0.001)$, respectively (Fig. $7 \mathrm{~b}, \mathrm{c})$. The overall clinicopathologic characteristics and clinical outcome of patients with IRE-HCCs are summarized in online supplementary Figure 2.

\section{Discussion}

Our results showed that IRE-HCCs demonstrate aggressive histopathologic features, including more prominent hypoxic and fibrotic tumor microenvironments and increased stemness compared to non-IRE-HCCs.

In our histopathologic analysis, the microvessels of IRE-HCCs consisted of higher proportions of sinusoid-like patterns and lower proportions of capillary-like patterns when compared to those of non-IRE-HCCs. Moreover, the microvascular density was positively correlated with the proportion of capillary-like microvascular patterns and inversely correlated with that of sinusoid-like microvascular patterns. IRE-HCCs showed lower microvascular density and poorer differentiation compared to non-IRE-HCCs. Microvascular density in HCC is known to be highly correlated with the degree of arterial enhancement on MRI or of peak enhancement on contrast-enhanced ultrasonography [26, 27]. The association between microvascular density and enhancement of HCC could be a possible explanation for the IRE-HCC to exhibit a large internal hypoenhancement area. There have been several reports of poorly differentiated HCCs exhibiting arterial phase hypoenhancement and low microvascular density compared to well or moderately differentiated HCCs $[25,28]$. Sinusoid-like microvascular pattern was also described as "vessels that encapsulate tumor cluster (VETC)" and it has been reported that this particular microvascular pattern could facilitate the microvascular invasion [29]. In line with the recently suggested biological mechanism of microvascular invasion, IRE and microvascular invasion are closely related in the present study and several previous studies [30,31]. Taken together, less enhancement of the central portion of the tumor in IRE-HCCs appears to be associated with low microvascular density and a sinusoid-like pattern, which are linked to poor differentiation, frequent microvascular invasion, and poor outcomes in HCC patients [21, 25].

In this study, IRE-HCCs also exhibited histopathologic characteristics indicative of a hypoxic microenvironment, such as lower microvascular density, a larger proportion of 


\section{Liver
Cancer}

\begin{tabular}{l|l}
\hline Liver Cancer 2019;8:24-40 \\
\hline DOI: 10.1159/000488540 & $\begin{array}{l}\text { @) 2018 S. Karger AG, Basel } \\
\text { www.karger.com/lic }\end{array}$ \\
\hline
\end{tabular}

tumor necrotic area, and frequent expression of the hypoxia-related marker CAIX. Hypoxia has been found to be involved in the maintenance of cancer stem cells, which are characterized by their ability to self-renew and propagate tumors, and play an important role in tumor maintenance and recurrence [32,33]. HCCs expressing stemness markers, such as K19 and EpCAM, are reportedly associated with poor prognosis and molecular signatures of aggressive biological behavior such as S2 (Hoshida et al. [34]) and G1 (Boyault et al. [35]) compared to HCCs that do not express these markers [18, 19, 34-38]. Rhee et al. [23] also recently reported a close association between the expression of the hypoxia marker CAIX and stemness markers including K19 and EpCAM in HCCs. In the present study, IRE-HCCs, which showed histopathologic characteristics indicative of a hypoxic microenvironment, expressed higher K19 and EpCAM levels, despite the lack of statistical significance for K19 level ( $p=$ 0.055 after Bonferroni correction).

The proportion of fibrous stroma was significantly higher in IRE-HCCs than in non-IREHCCs in our study. Cancer-associated fibroblasts not only produce fibrous stroma but also play an important role in cancer progression through paracrine interaction with epithelial cancer cells $[39,40]$. Notably, HCCs with stemness were reported to have more fibrous stroma compared to those without, suggesting an interaction between cancer stem cells and fibrous tumor stroma [34]. Scirrhous HCC, a rare variant of the disease that is characterized by abundant fibrous stromal components comprising 30-50\% of the tumor area, was reported to show increased expression of stemness markers including K19 and EpCAM, as well as upregulation of epithelial-to-mesenchymal transition- and invasion-related genes, compared to typical HCCs without fibrous stroma [20,41]. Jeong et al. [14] previously reported that HCCs with abundant fibrous stroma or K19 positivity tend to show IRE on gadoxetateenhanced MRI.

One of the pathologic characteristics of IRE-HCCs is the macrotrabecular pattern, which has been demonstrated to be closely correlated with microvascular characteristics and is closely associated with sinusoid-like microvascular patterns and low microvascular density $[24,25]$. Recently, HCCs with a macrotrabecular pattern were also reported to belong to the S2 signature subclass described by Hoshida and colleagues [18, 34], and were correlated with the activation of the YAP oncogene and with the expression of the stemness markers K19 and EpCAM. Moreover, macrotrabecular-massive HCCs were found to belong to the G3 signature subclass described by Boyault et al. [34], which is known to be an aggressive molecular subtype of HCC, and were correlated with poor survival, vascular invasion, TP53 mutation, and FGF19 amplification [19, 34]. Therefore, IREs might represent distinct histopathologic and molecular characteristics of HCC via their association with the macrotrabecular pattern.

Cases in which the two reviewers reported inconsistent enhancement patterns were found to have mixed histopathologic components or traits that were intermediate between IRE-HCCs and non-IRE-HCCs. Therefore, the discrepancies in radiologic classifications were likely a consequence of these mixed or intermediate histopathologic features rather than the reviewers' subjectivities. One interesting result of this study is that 3 cases of HCC with intermediate enhancement patterns showed a nodule-in-nodule appearance, which is thought to be a process by which HCCs progress [42], on both MRI and histopathologic analyses. In these cases, the outer nodules showed hyperenhancement and capillary-like microvascular patterns, whereas the inner nodules exhibited hypoenhancement and sinusoid-like microvascular patterns.

This study aimed to seek a histopathologic explanation for the results of our previous HCC studies in which IRE was an independent imaging feature associated with poor prognosis after curative resection $[3,5]$. We found that the histopathologic features of IRE-HCCs included sinusoid-like microvascular pattern, macrotrabecular pattern, a high proportion of 
tumor necrosis and fibrous stroma, and expression of stemness markers, which have been reported as characteristics of aggressive and poor prognostic HCCs [20, 21, 23, 25, 43]. Therefore, IRE might be a promising noninvasive biomarker for poor prognostic HCCs showing distinct pathologic features. Actually, it is difficult to differentiate by imaging alone between atypical HCCs with IRE and other primary malignancies with more aggressive behavior including cholangiocarcinoma, combined hepatocellular-cholangiocarcinoma, or the sarcomatoid variant of HCC due to their similar imaging features. Therefore, a primary hepatic tumor with IRE on imaging study is considered to be related to aggressive behavior, and further workup including biopsy is required for definite diagnosis.

In the current study, $5.3 \%$ of the 431 resected HCCs were initially selected as IRE-HCCs for histopathologic analysis. This proportion is similar to that in our recent study in which $4.9 \%$ of 225 HCCs showed IRE and were thus categorized as LR-M [5], but lower than that in our earlier study in which $11.8 \%$ of the 187 HCCs showed IRE [3]. A likely reason for this discrepancy is our strict criteria for evaluation of IRE in the current study; we considered IRE to be positive only when both of the two reviewers agreed on its presence. In contrast, in our initial study [3], we were less conservative, and IRE was considered to be positive when one of the two reviewers, not both of them, determined its presence. Indeed, even in the current study, the second radiologist was less conservative in determining the presence of IRE and classified $9.3 \%$ as IRE-HCCs, which is a similar proportion to that observed in our initial study. Furthermore, the proportions of IRE-HCCs in our studies were likely to be underestimated compared to its real incidence, because we only included resected tumors; aggressive tumors showing IRE are more likely to be found in advanced stages and thus to be excluded from curative resection.

Our study had some limitations. First, a single radiologist enrolled the patients by retrospectively reviewing MRI data, which may have led to selection bias. To minimize this, a second radiologist performed an independent review of the MRI data; additionally, detailed histopathologic analyses were performed for the cases in which the two reviewers did not agree. Second, we did not perform our histopathologic analysis in all cases of non-IRE-HCCs. However, we included randomly selected non-IRE-HCCs cases, more than twice as many as IRE-HCC, which we thought was sufficient for the comparison of histologic features. Furthermore, the imaging and survival analyses were performed on the entire study cohort.

In conclusion, IRE-HCCs exhibit aggressive histopathologic features, including a more hypoxic and fibrotic tumor microenvironment and increased stemness compared to non-IREHCCs. Our results highlight the value of IRE as a biomarker for aggressive HCC that can be assessed noninvasively through imaging.

\section{Acknowledgments}

This study was supported by a grant from the National R\&D Program for Cancer Control, Ministry of Health and Welfare, South Korea (grant 1520160 to M.-J.K.) and grants from the National Research Foundation of Korea (NRF) funded by the Korean government (MSIP) (grants NRF-2011-0030086, NRF2016M3A9D5A01952416, NRF-2017R1A2B4005871, and NRF-2017M3A9B6061512 to Y.N.P.).

\section{Disclosure Statement}

Myeong-Jin Kim has received a grant from Bayer HealthCare, which is not related to the present study. The remaining authors have no conflicts of interest to declare. 
Rhee et al.: Aggressive Pathologic Features of HCC with Rim-Like Arterial Enhancement

\section{Author Contributions}

Hyungjin Rhee and Chansik An were involved in the conception and design, development of methodology, acquisition of data, analysis and interpretation of data, and writing the manuscript. Hye-Young Kim and Jeong Eun Yoo were engaged in the acquisition of data, analysis of data, review of the manuscript, and technical support. Myeong-Jin Kim and Young Nyun Park carried out the conception and design, acquisition of data, analysis and interpretation of data, writing and review of the manuscript, study supervision, and financing of the study.

\section{References}

1 Forner A, Vilana R, Ayuso C, Bianchi L, Sole M, Ayuso JR, Boix L, Sala M, Varela M, Llovet JM, Bru C, Bruix J: Diagnosis of hepatic nodules $20 \mathrm{~mm}$ or smaller in cirrhosis: prospective validation of the noninvasive diagnostic criteria for hepatocellular carcinoma. Hepatology 2008;47:97-104.

2 Marrero JA, Hussain HK, Nghiem HV, Umar R, Fontana RJ, Lok AS: Improving the prediction of hepatocellular carcinoma in cirrhotic patients with an arterially-enhancing liver mass. Liver Transpl 2005;11:281-289.

3 An C, Kim DW, Park YN, Chung YE, Rhee H, Kim MJ: Single hepatocellular carcinoma: preoperative MR imaging to predict early recurrence after curative resection. Radiology 2015;276:433-443.

4 Kawamura Y, Ikeda K, Seko Y, Hosaka T, Kobayashi M, Saitoh S, Kumada H: Heterogeneous type 4 enhancement of hepatocellular carcinoma on dynamic CT is associated with tumor recurrence after radiofrequency ablation. AJR Am J Roentgenol 2011;197:W665-W673.

5 An C, Park S, Chung YE, Kim DY, Kim SS, Kim MJ, Choi JY: Curative resection of single primary hepatic malignancy: liver imaging reporting and data system category LR-M portends a worse prognosis. AJR Am J Roentgenol 2017;209:576-583.

6 Lee JH, Chung GE, Yu SJ, Hwang SY, Kim JS, Kim HY, Yoon JH, Lee HS, Yi NJ, Suh KS, Lee KU, Jang JJ, Kim YJ: Longterm prognosis of combined hepatocellular and cholangiocarcinoma after curative resection comparison with hepatocellular carcinoma and cholangiocarcinoma. J Clin Gastroenterol 2011;45:69-75.

7 Lee WS, Lee KW, Heo JS, Kim SJ, Choi SH, Kim YI, Joh JW: Comparison of combined hepatocellular and cholangiocarcinoma with hepatocellular carcinoma and intrahepatic cholangiocarcinoma. Surg Today 2006;36:892897.

8 Bosch FX, Ribes J, Diaz M, Cleries R: Primary liver cancer: worldwide incidence and trends. Gastroenterology 2004;127:S5-S16.

9 Cha DI, Jang KM, Kim SH, Kang TW, Song KD: Liver imaging reporting and data system on CT and gadoxetic acid-enhanced MRI with diffusion-weighted imaging. Eur Radiol 2017;27:4394-4405.

10 Kierans AS, Leonardou P, Hayashi P, Brubaker LM, Elazzazi M, Shaikh F, Semelka RC: MRI findings of rapidly progressive hepatocellular carcinoma. Magn Reson Imaging 2010;28:790-796.

11 Kim BK, Kim KA, An C, Yoo EJ, Park JY, Kim DY, Ahn SH, Han KH, Kim SU, Kim MJ: Prognostic role of magnetic resonance imaging vs. computed tomography for hepatocellular carcinoma undergoing chemoembolization. Liver Int 2015;35:1722-1730.

12 Kang TW, Rhim H, Lee J, Song KD, Lee MW, Kim YS, Lim HK, Jang KM, Kim SH, Gwak GY, Jung SH: Magnetic resonance imaging with gadoxetic acid for local tumour progression after radiofrequency ablation in patients with hepatocellular carcinoma. Eur Radiol 2016;26:3437-3446.

13 Kim SH, Lim HK, Lee WJ, Choi D, Park CK: Scirrhous hepatocellular carcinoma: comparison with usual hepatocellular carcinoma based on CT - pathologic features and long-term results after curative resection. Eur J Radiol 2009;69:123-130.

14 Jeong HT, Kim MJ, Kim YE, Park YN, Choi GH, Choi JS: MRI features of hepatocellular carcinoma expressing progenitor cell markers. Liver Int 2012;32:430-440.

15 Kawamura Y, Ikeda K, Hirakawa M, Yatsuji H, Sezaki H, Hosaka T, Akuta N, Kobayashi M, Saitoh S, Suzuki F, Suzuki Y, Arase Y, Kumada H: New classification of dynamic computed tomography images predictive of malignant characteristics of hepatocellular carcinoma. Hepatol Res 2010;40:1006-1014.

16 Mitchell DG, Bruix J, Sherman M, Sirlin CB: LI-RADS (Liver Imaging Reporting and Data System): summary, discussion, and consensus of the LI-RADS Management Working Group and future directions. Hepatology 2015;61:1056-1065.

17 An C, Rakhmonova G, Choi JY, Kim MJ: Liver imaging reporting and data system (LI-RADS) version 2014: understanding and application of the diagnostic algorithm. Clin Mol Hepatol 2016;22:296-307.

18 Tan PS, Nakagawa S, Goossens N, Venkatesh A, Huang T, Ward SC, Sun X, Song WM, Koh A, Canasto-Chibuque C, Deshmukh M, Nair V, Mahajan M, Zhang B, Fiel MI, Kobayashi M, Kumada H, Hoshida Y: Clinicopathological indices to predict hepatocellular carcinoma molecular classification. Liver Int 2016;36:108-118.

19 Calderaro J, Couchy G, Imbeaud S, Amaddeo G, Letouze E, Blanc JF, Laurent C, Hajji Y, Azoulay D, Bioulac-Sage P, Nault JC, Zucman-Rossi J: Histological subtypes of hepatocellular carcinoma are related to gene mutations and molecular tumour classification. J Hepatol 2017;67:727-738. 
20 Seok JY, Na DC, Woo HG, Roncalli M, Kwon SM, Yoo JE, Ahn EY, Kim GI, Choi JS, Kim YB, Park YN: A fibrous stromal component in hepatocellular carcinoma reveals a cholangiocarcinoma-like gene expression trait and epithelial-mesenchymal transition. Hepatology 2012;55:1776-1786.

21 Chen ZY, Wei W, Guo ZX, Lin JR, Shi M, Guo RP: Morphologic classification of microvessels in hepatocellular carcinoma is associated with the prognosis after resection. J Gastroenterol Hepatol 2011;26:866-874.

22 McDonald PC, Winum JY, Supuran CT, Dedhar S: Recent developments in targeting carbonic anhydrase IX for cancer therapeutics. Oncotarget 2012;3:84-97.

23 Rhee H, Nahm JH, Kim H, Choi GH, Yoo JE, Lee HS, Koh MJ, Park YN: Poor outcome of hepatocellular carcinoma with stemness marker under hypoxia: resistance to transarterial chemoembolization. Mod Pathol 2016;29: 1038-1049.

24 Tanigawa N, Lu C, Mitsui T, Miura S: Quantitation of sinusoid-like vessels in hepatocellular carcinoma: its clinical and prognostic significance. Hepatology 1997;26:1216-1223.

25 Murakami K, Kasajima A, Kawagishi N, Ohuchi N, Sasano H: Microvessel density in hepatocellular carcinoma: prognostic significance and review of the previous published work. Hepatol Res 2015;45:1185-1194.

26 Wang Z, Tang J, An L, Wang W, Luo Y, Li J, Xu J: Contrast-enhanced ultrasonography for assessment of tumor vascularity in hepatocellular carcinoma. J Ultrasound Med 2007;26:757-762.

27 Wang B, Gao ZQ, Yan X: Correlative study of angiogenesis and dynamic contrast-enhanced magnetic resonance imaging features of hepatocellular carcinoma. Acta Radiol 2005;46:353-358.

28 Lee JH, Lee JM, Kim SJ, Baek JH, Yun SH, Kim KW, Han JK, Choi BI: Enhancement patterns of hepatocellular carcinomas on multiphasicmultidetector row CT: comparison with pathological differentiation. Br J Radiol 2012;85:e573-583.

29 Fang JH, Zhou HC, Zhang C, Shang LR, Zhang L, Xu J, Zheng L, Yuan Y, Guo RP, Jia WH, Yun JP, Chen MS, Zhang Y, Zhuang SM: A novel vascular pattern promotes metastasis of hepatocellular carcinoma in an epithelialmesenchymal transition-independent manner. Hepatology 2015;62:452-465.

30 Lee S, Kim SH, Lee JE, Sinn DH, Park CK: Preoperative gadoxetic acid-enhanced MRI for predicting microvascular invasion in patients with single hepatocellular carcinoma. J Hepatol 2017;67:526-534.

31 Renzulli M, Brocchi S, Cucchetti A, Mazzotti F, Mosconi C, Sportoletti C, Brandi G, Pinna AD, Golfieri R: Can current preoperative imaging be used to detect microvascular invasion of hepatocellular carcinoma? Radiology 2016;279:432-442.

32 Yamashita T, Kaneko S: Orchestration of hepatocellular carcinoma development by diverse liver cancer stem cells. J Gastroenterol 2014;49:1105-1110.

33 Yamashita T, Wang XW: Cancer stem cells in the development of liver cancer. J Clin Invest 2013;123:19111918.

34 Hoshida Y, Nijman SM, Kobayashi M, Chan JA, Brunet JP, Chiang DY, Villanueva A, Newell P, Ikeda K, Hashimoto M, Watanabe G, Gabriel S, Friedman SL, Kumada H, Llovet JM, Golub TR: Integrative transcriptome analysis reveals common molecular subclasses of human hepatocellular carcinoma. Cancer Res 2009;69:7385-7392.

35 Boyault S, Rickman DS, de Reynies A, Balabaud C, Rebouissou S, Jeannot E, Herault A, Saric J, Belghiti J, Franco D, Bioulac-Sage P, Laurent-Puig P, Zucman-Rossi J: Transcriptome classification of HCC is related to gene alterations and to new therapeutic targets. Hepatology 2007;45:42-52.

36 Kim H, Choi GH, Na DC, Ahn EY, Kim GI, Lee JE, Cho JY, Yoo JE, Choi JS, Park YN: Human hepatocellular carcinomas with "Stemness"-related marker expression: keratin 19 expression and a poor prognosis. Hepatology 2011;54:1707-1717.

37 Guo Z, Li LQ, Jiang JH, Ou C, Zeng LX, Xiang BD: Cancer stem cell markers correlate with early recurrence and survival in hepatocellular carcinoma. World J Gastroenterol 2014;20:2098-2106.

38 Chan AW, Tong JH, Chan SL, Lai PB, To KF: Expression of stemness markers (CD133 and EpCAM) in prognostication of hepatocellular carcinoma. Histopathology 2014;64:935-950.

39 Gascard P, Tlsty TD: Carcinoma-associated fibroblasts: orchestrating the composition of malignancy. Genes Dev 2016;30:1002-1019.

40 Plaks V, Kong N, Werb Z: The cancer stem cell niche: how essential is the niche in regulating stemness of tumor cells? Cell Stem Cell 2015;16:225-238.

41 Kurogi M, Nakashima O, Miyaaki H, Fujimoto M, Kojiro M: Clinicopathological study of scirrhous hepatocellular carcinoma. J Gastroenterol Hepatol 2006;21:1470-1477.

42 Kudo M: Multistep human hepatocarcinogenesis: correlation of imaging with pathology. J Gastroenterol 2009; 44(suppl 19):112-118.

43 Ziol M, Pote N, Amaddeo G, Laurent A, Nault JC, Oberti F, Costentin C, Michalak S, Bouattour M, Francoz C, Pageaux GP, Ramos J, Decaens T, Luciani A, Guiu B, Vilgrain V, Aube C, Derman J, Charpy C, Zucman-Rossi J, Barget N, Seror O, Ganne-Carrie N, Paradis V, Calderaro J: Macrotrabecular-massive hepatocellular carcinoma: a distinctive histological subtype with clinical relevance. Hepatology 2017, Epub ahead of print. 